\title{
A Severe Case of Molluscum Contagiosum Immune Reconstitution Inflammatory Syndrome in a Patient with Human Immunodeficiency Virus
}

\author{
Nida Siddiqui ${ }^{1}$, Brett S Mansfield ${ }^{1}$, Nine-Paula OImesdahl ${ }^{2}$, Peter Swart ${ }^{2}$, Jeremy Nel $^{3}$ \\ ${ }^{1}$ Dept. of Internal Medicine, School of Clinical Medicine, Faculty of Health Sciences, University of the Witwatersrand, Johannesburg, South Africa \\ ${ }^{2}$ Division of Anatomical Pathology, Dept. of Pathology, National Health Laboratory Service and University of the Witwatersrand, Johannesburg, South Africa \\ ${ }^{3}$ Division of Infectious Diseases, Dept. of Internal Medicine, School of Clinical Medicine, Faculty of Health Sciences, University of the Witwatersrand, \\ Johannesburg, South Africa
}

Received: 09/12/2021

Accepted: $14 / 12 / 2021$

Published: $11 / 01 / 2022$

How to cite this article: Siddiqui N, Mansfield BS, Olmesdahl NP, Swart P, Nel J. A severe case of Molluscum contagiosum immune reconstituition inflammatory syndrome in a patient with human immunodeficiency virus. EJCRIM 2022;9: doi:10.12890/2022_003115.

Conflicts of Interests: The authors declare there are no competing interests.

This article is licensed under a Commons Attribution Non-Commercial 4.0 License

\section{ABSTRACT}

Paradoxical immune reconstitution inflammatory syndrome (IRIS) in human immunodeficiency virus (HIV)-positive patients initiating antiretroviral treatment (ART) is caused by restored immunity to specific antigens, resulting in worsening of a pre-existing infection. Molluscum contagiosum (MC) is commonly noted in HIV-positive individuals but ART alone is usually sufficient to bring about resolution. We present a rare case of severe MC-IRIS that worsened despite immune reconstitution.

\section{LEARNING POINTS}

- Molluscum contagiosum is a common opportunistic infection which can have severe manifestations in immunocompromised individuals.

- Antiretroviral treatment alone is usually sufficient to clear the infection, however refractory cases can persist despite immune reconstitution.

- Failure to improve or worsening immune reconstitution inflammatory syndrome should raise suspicion for additional immunological dysfunction.

- Surgery, cytodestructive therapies and chemotherapeutic agents can be considered in extensive, persistent disease.

\section{KEYWORDS}

Immune reconstitution inflammatory syndrome, molluscum contagiosum, human immunodeficiency virus, MC-IRIS

\section{CASE DESCRIPTION}

A 57-year-old man infected with HIV, with a CD4 count of 304 cells/ $\mu$ l and a suppressed viral load, was referred to our institution with an extensive facial rash. He had first noticed two small skin lesions 8 months previously. Shortly thereafter he was diagnosed with HIV and initiated on first-line antiretroviral treatment (ART), comprising tenofovir, emtricitabine and efavirenz. The skin rash worsened 2-3 months following the initiation of ART. He had no other comorbidities and no history of opportunistic infections.

Examination was notable for 2-3 mm painless nodules and umbilicated papules found over his face, ears, neck and chest. These lesions were raised, dome-shaped and flesh-coloured with central pitting and a verrucous surface. Giant lesions (>1 cm) were also seen (Fig. 1). There was no involvement of the mucous membranes, palms or soles of the feet. Initial blood results revealed a mild normocytic anaemia and mild acute kidney injury which subsequently resolved (Table 1). 

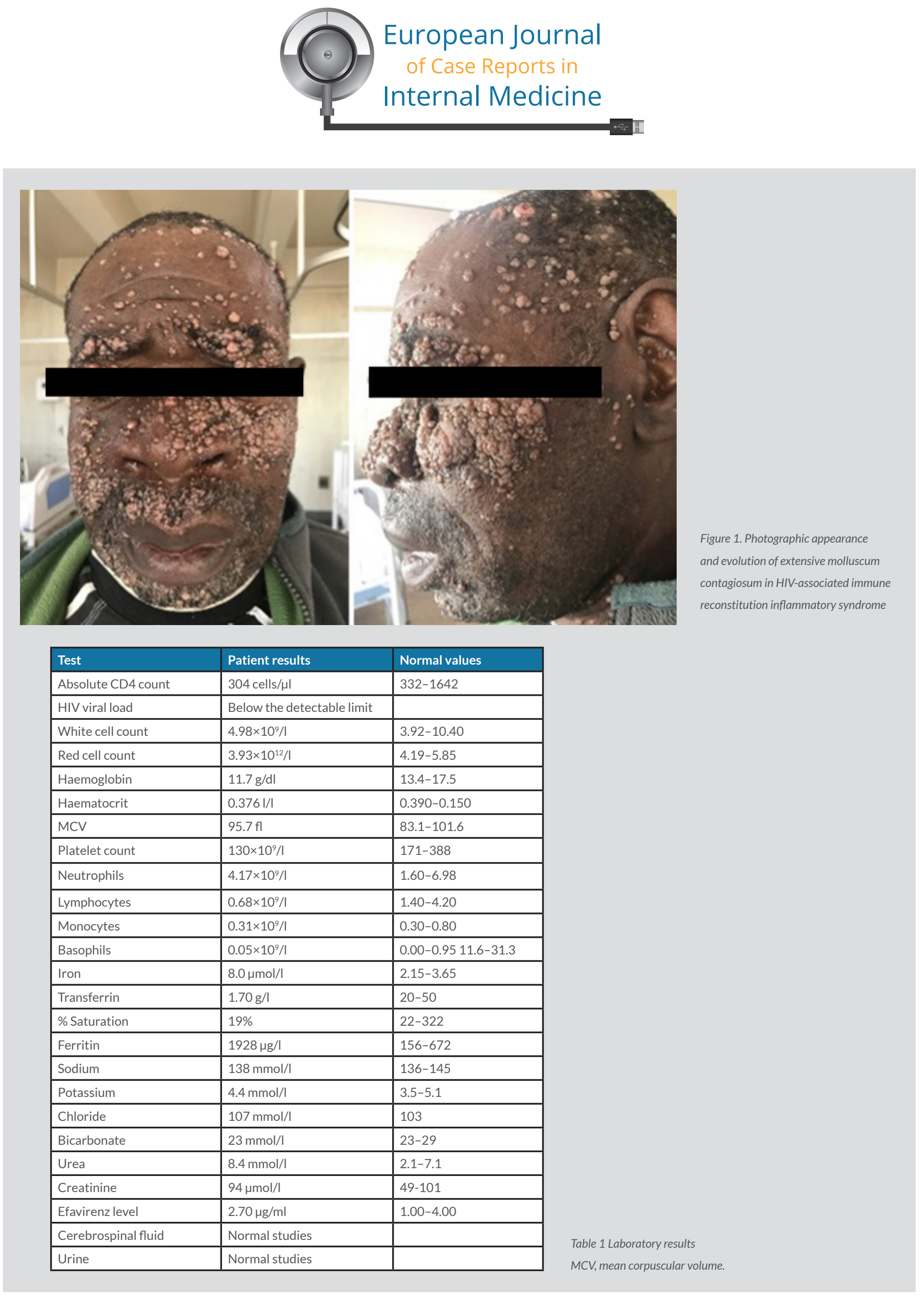
Differential diagnoses which were considered at this point included molluscum contagiosum (MC), human papilloma virus and cutaneous cryptococcosis. A skin biopsy revealed large cytoplasmic eosinophilic inclusion bodies (Henderson-Paterson bodies) which are pathognomonic for MC ${ }^{[1]}$ (Figs. 2 and 3).

Our patient was discharged generally well with a multidisciplinary follow-up plan which included outpatient visits to dermatology, infectious diseases and psychology. He unfortunately experienced a sudden deterioration in his health just before his first follow-up appointment and died unexpectedly.
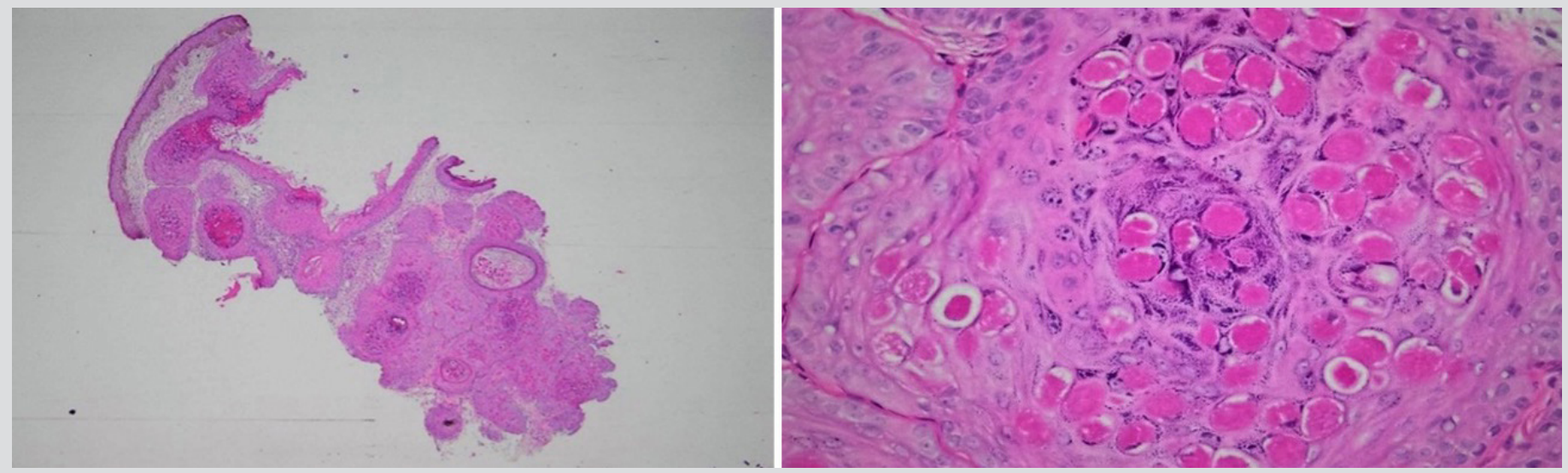

Figure 2. (Left image) A skin biopsy including epidermis and dermis showing an inverted lobule of hyperplastic squamous epithelium extending into the dermis (H\&E stain $\times 20$ magnification). (Right image) Large eosinophilic viral inclusion bodies also known as Henderson-Paterson or molluscum bodies (H\&E ×40 magnification)

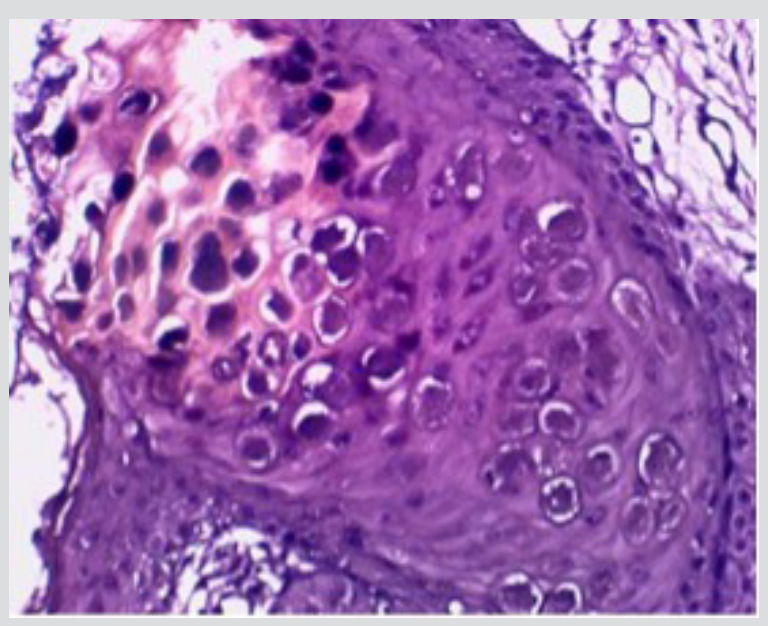

Figure 3. Giemsa stain highlighting molluscum bodies

\section{DISCUSSION}

Immune reconstitution inflammatory syndrome (IRIS) is an excessive immune reaction to a pre-existing infection following the initiation of ART [2]. The pathogens most frequently associated with IRIS are Mycobacterium tuberculosis (MTB), non-tuberculous mycobacteria, cytomegalovirus (CMV) and Cryptococcus neoformans. More than half of the individuals who develop IRIS will present with a cutaneous manifestation, most commonly varicella zoster and herpes simplex virus ${ }^{[3]}$. Less common aetiologies include Pneumocystisjirovecii pneumonia, Toxoplasma gondii, hepatitis $\mathrm{B}$ and $\mathrm{C}$ virus, and rarely, $\mathrm{MC}$ virus ${ }^{[2]}$.

$\mathrm{MC}$ is usually a benign, self-limiting viral infection manifesting with fewer than 20 small, discrete, waxy, skin-coloured dome-shaped papules which are 3-5 mm in diameter ${ }^{[1]}$. The virus is uniquely adapted to the skin. It infects cells in the epidermis and enhances mitosis and the disruption of epidermal cell differentiation ${ }^{[4]}$. It survives and proliferates by inhibiting tumour necrosis factor-alpha-induced apoptosis of virally infected cells. Intact cell-mediated immunity, in the form of CD4 T-lymphocytes and Langerhans cells, plays an important role in its clearance ${ }^{[4]}$. The virus does not cross the basement membrane, thus escaping most of the body's immune surveillance, preventing the development of the innate immune response and contributing to the persistence of the lesions ${ }^{[4]}$. 
The presence of MC may indicate immunosuppression. Individuals with HIV or taking immunosuppressive therapy may experience more widespread, persistent and/or recurrent infections ${ }^{[1]}$. MC is common and occurs in $5-20 \%$ of HIV-infected patients, but molluscum contagiosum-immune reconstitution inflammatory syndrome (MC-IRIS) is rare ${ }^{[1]}$.

Treating HIV infection by ART alone is generally sufficient in cases of MC-IRIS ${ }^{[1,2,4]}$. Further treatment options include surgery (curettage, electrodessication, cryotherapy with liquid nitrogen, and laser surgery), cytodestructive methods (cantharidin, iodine, lactic acid, phenol, salicylic acid, silver nitrate, isotretinoin and trichloroacetic acid), antiviral drugs (cidofovir) and chemotherapy (interferon and imiquimod ${ }^{[1,4]}$. Although no longer recommended as conventional therapy, topical imiquimod has led to successful treatment of refractory MC infection in HIV-infected individuals ${ }^{[1]}$. Cidofovir, an antiviral agent used to treat CMV-retinitis in patients with advanced HIV, has shown activity against the pox-virus family, but renal toxicity is a concern ${ }^{[1,4]}$. Pulse dye laser therapy has also had promising results in the treatment of refractory MC. However, both topical cidofovir and pulse dye laser therapy are costly and not available in resource-limited settings. Furthermore, there is a paucity of data regarding the efficacy of these two therapies and more prospective studies are needed ${ }^{[1]}$.

Our patient presented with the hallmark features of paradoxical IRIS. The extent of MC infection worsened dramatically after ART initiation. A high initial burden of disease, a low nadir CD4 count, and high baseline viral load are well established risk factors for IRIS reactions, and although baseline clinical data were not available for this patient, it is likely that all three factors contributed in this case. Nonetheless, the clinical worsening seen in MC-IRIS is usually temporary, and gradually improves with time. Thus, it is possible that our patient had additional immunological dysfunction. Incomplete immune reconstitution and residual inflammation may have contributed in this case. This can occur as a result of an imbalance between innate and adaptive immunity and may lead to an increase in morbidity and mortality ${ }^{[5]}$.

Disseminated MC is more common in HIV-infected individuals with low CD4 counts but usually resolves after immunological recovery of the CD4 count to greater than 200 cells/ $\mu$ following ART initiation. Our patient presented with a paradoxical MC-IRIS that failed to improve on ART despite a CD4 count of more than 300 cells/ $\mu$ l and a suppressed viral load.

\section{REFERENCES}

1. Robinson G, Townsend S, Jahnke MN. Molluscum contagiosum: review and update on clinical presentation, diagnosis, risk, prevention, and treatment. Curr Derm Rep 2020;9:83-92.

2. Yang H-S, Li C-W, Hsieh F-N, Liu C-H, Lee JY-Y, Yang C-C. Molluscum contagiosum-associated immune reconstitution inflammatory syndrome in human immunodeficiency virus infection. Dermatologica Sinica 2016;34(4):196-9.

3. Osei-Sekyere B, Karstaedt AS. Immune reconstitution inflammatory syndrome involving the skin. Clin Exp Dermatol 2010;35(5):477-81.

4. Chen X, Anstey AV, Bugert JJ. Molluscum contagiosum virus infection. Lancet Infect Dis 2013;13(10):877-88.

5. Wilson EM, Sereti I. Immune restoration after antiretroviral therapy: the pitfalls of hasty or incomplete repairs. Immunol Rev 2013;254(1):343-54. 